\title{
Marked genetic differences between BRAF and NRAS mutated primary melanomas as revealed by array comparative genomic hybridization Viktória Lázár ${ }^{a}$, Szilvia Ecsedi ${ }^{a, c}$, Laura Vizkeleti ${ }^{a}$, Zsuzsa Rákosy ${ }^{a, c}$, Gábor Boross ${ }^{\mathrm{d}}$, Balázs Szappanos ${ }^{\mathrm{d}}$, Ágnes Bégány ${ }^{\mathrm{b}}$, Gabriella Emri ${ }^{\mathrm{b}}$, Róza Ádány ${ }^{\mathrm{a}, \mathrm{c}}$ and Margit Balázs ${ }^{\mathrm{a}, \mathrm{c}}$
}

Somatic mutations of BRAF and NRAS oncogenes are thought to be among the first steps in melanoma initiation, but these mutations alone are insufficient to cause tumor progression. Our group studied the distinct genomic imbalances of primary melanomas harboring different BRAF or NRAS genotypes. We also aimed to highlight regions of change commonly seen together in different melanoma subgroups. Array comparative genomic hybridization was performed to assess copy number changes in 47 primary melanomas. BRAF and NRAS were screened for mutations by melting curve analysis. Reverse transcription polymerase chain reaction and fluorescence in situ hybridization were performed to confirm the array comparative genomic hybridization results. Pairwise comparisons revealed distinct genomic profiles between melanomas harboring different mutations. Primary melanomas with the BRAF mutation exhibited more frequent losses on 10q23-q26 and gains on chromosome 7 and 1q23-q25 compared with melanomas with the NRAS mutation. Loss on the 11q23-q25 sequence was found mainly in conjunction with the NRAS mutation. Primary melanomas without the BRAF or the NRAS mutation showed frequent alterations in chromosomes 17 and 4. Correlation analysis revealed chromosomal alterations that coexist more often in these tumor subgroups. To find classifiers for BRAF mutation, random forest analysis was

\section{Introduction}

Skin cancer is the most widespread malignancy in most countries and although melanoma represents only a small subset, it is one of the most dangerous cutaneous neoplasms that arise from pigmented cells. As soon as the first distant metastasis appears, the disease becomes one of the most aggressive and chemoresistant tumors. Even though the early recognition of cutaneous melanoma has improved, the mortality rate has not changed and has shown stabilization in Australia, USA, and Europe [1-3]. Thus, it is becoming a major public health problem, which requires efforts to determine the genetic and environmental factors of melanoma genesis and progression [4]. Different subtypes of the disease represent diverse entities, as there are marked differences in their biological behavior, and it is suggested that this morphologic heterogeneity originates from underlying genetics, leading to used. Fifteen candidates emerged with $87 \%$ prediction accuracy. Signaling interactions between the EGF/ MAPK-JAK pathways were observed to be extensively altered in melanomas with the BRAF mutation. We found marked differences in the genetic pattern of the BRAF and NRAS mutated melanoma subgroups that might suggest that these mutations contribute to malignant melanoma in conjunction with distinct cooperating oncogenic events. Melanoma Res 00:000-000 (c) 2012 Wolters Kluwer Health | Lippincott Williams \& Wilkins.

Melanoma Research 2012, 00:000-000

Keywords: array comparative genomic hybridization, BRAF mutation, NRAS mutation, primary melanoma, signaling pathway

${ }^{a}$ Department of Preventive Medicine, Faculty of Public Health, ${ }^{\text {b Department }}$ of Dermatology, Faculty of Medicine, Medical and Health Science Center, ${ }^{\mathrm{C}}$ Public Health Research Group of the Hungarian Academy of Sciences, University of Debrecen, Debrecen, Hungary and dEvolum Syelogy Group, Institute of Biochemistry, Biological Research Centre, Szeged, Hungary

Correspondence to Margit Balázs, PhD, DSc, Division of Biomarker Analysis, Department of Preventive Medicine, Faculty of Public Health, Medical and Health Science Center, University of Debrecen, Kassai str. 26, $\mathrm{H}-4028$ Debrecen, Hungary

Tel:/fax: + 3652417267 ;

e-mail: balazs.margit@sph.unideb.hu

Received 11 October 2011 Accepted 22 February 2012

diverse pathways of tumor development and progression [5]. For example, activation of the mitogen-activated protein kinase (EGF/MAPK) pathway through mutations in $B R A F$ or NRAS and loss of PTEN is a compulsory event in the subgroup of melanoma that develop in skin that was exposed to intermittent UV radiation [6].

Until recently, histopathology has been the main standard for the diagnosis of melanoma, but there are already some reports showing that genetic data provided by comparative genomic hybridization $(\mathrm{CGH})$ can yield helpful diagnostic information in cases that are ambiguous on the basis of histopathologic assessment [7]. The recent development of high-resolution molecular biological techniques has advanced our ability to detect genetic alterations in the entire genome. The largest outcome study combined the results of mutational analysis of $B R A F$ and $N R A S$ 
oncogenes with array CGH (aCGH) data in 126 melanomas from individuals with varying UV exposure and has yielded interesting information: several marked differences in aberrant genomic regions and in the frequencies of $B R A F$ and NRAS mutations were found in the groups in which the degree of sun exposure differed [5]. These findings indicated distinct genetic pathways in the development of melanoma that could affect the design of targeted therapeutic interventions in the future. The study also revealed that melanoma is a heterogenous disease with an unpredictable clinical course. Using tilingresolution bacterial artificial chromosome (BAC) aCGH, another study observed discrete copy number alterations associated with mutations in various melanoma genes, including BRAF, NRAS, PTEN, and TP53, in 47 different melanoma cell lines. Moreover, two recent landmark studies investigated the distinct genome-wide alterations in DNA copy number associated with $B R A F$ or $N R A S$ mutation status in 43 primary human melanomas and several melanoma cell lines [8-10]. In addition, we recently found that coamplification of candidate oncogenes in the $11 \mathrm{q} 13$ region with either the $B R A F$ or the NRAS mutation might be more important for prognosis than these alterations alone [11]. These previous reports suggested that even though both $B R A F$ and NRAS function as key molecules along the EGF/MAPK pathway, they may cooperate with different oncogenic events during melanoma development.

In this study, aCGH was used to assess gene copy number changes in 47 primary cutaneous melanomas. Thereafter, these lesions were screened for the most common $B R A F$ and NRAS mutations found in melanoma to establish distinct mutation subgroups of the disease such as $B R A F$ mutated or NRAS mutated or wild-type (WT) (assigned as BRAF $^{\text {mut }}$, BRAF $^{\mathrm{WT}}$ and NRAS ${ }^{\text {mut }}$, NRAS ${ }^{\mathrm{WT}}$, respectively) for both of these oncogenes. It is well known that activating mutations in $B R A F$ and $N R A S$ are, so far, the most common single mutations detected in melanoma and the majority of benign nevi, but it is also clear that isolated mutations are not sufficient to initiate human melanoma in vivo. As the significance of BRAF and NRAS mutations in melanoma has remained unclear, the two major objectives of our investigation were: (a) to elucidate chromosomal regions that differ in copy number between these genetically different melanomas and (b) to examine the correlations between these regions (which covers important onco-suppressor and/or tumor-suppressor genes) to explore whether some of them act together in generating group differences. Another major focus of our study was to explore a possible set of gene copy number alterations that have significant impacts on dysregulation of the EGF/MAPK pathway along with the $B R A F$ mutation. Furthermore, we obtained the entire set of a signaling pathway data cataloged in a novel database and estimated the copy number changes of each of these pathway genes using the closest BAC clone to investigate the large-scale modifications in signaling interactions (later referred as cross-talks) between and within different pathways in a series of primary melanomas.

\section{Materials and methods}

\section{Tumor samples and DNA isolation}

Tissue samples were obtained from 47 patients who were diagnosed with primary cutaneous melanoma and subsequently underwent surgery between 1995 and 2006 at the University of Debrecen, Medical and Health Science Center, Department of Dermatology, Hungary. The study was approved by the Regional and Institutional Ethics Committee, Medical and Health Science Center, University of Debrecen, and was conducted according to regulations. Tumor diagnosis was made on the basis of formalin-fixed paraffin embedded tissue sections using hematoxylin and eosin staining. A primary melanoma tissue that was used to extract DNA for aCGH was considered suitable for study if the proportion of tumor cells was higher than $70 \%$. Melanoma tumor staging was determined according to the current tumor node metastasis staging system [12].

The follow-up period was 4 years. Table 1 summarizes the clinicopathological data. Genomic DNA was obtained from frozen tissue samples using the DNeasy kit (Quiagen, Hilden, Germany) and the G-spin Genomic DNA Extraction Kit (Macherey-Nagel, Düren, Germany) according to the manufacturers' instructions.

\section{Array comparative genomic hybridization experiments} aCGH experiments were conducted on HumArray 3.1 obtained from the University of California, San Francisco Cancer Center Array Core, as described before [13]. This array contains $2464 \mathrm{BAC}$ and $\mathrm{P} 1$ clones, printed in triplicate and covering the genome at roughly $1.4 \mathrm{Mb}$ resolution. Hybridization and imaging setup were performed as previously described [14]. The acquired microarray images were analyzed by Spot and Sproc software (UCSF Comprehensive Cancer Center, University of California, San Fransico, California, USA) [15]. DNA spots were automatically segmented, local background was subtracted, and the intensity ratio of the two dyes for each spot was calculated by $\log _{2}$-transformed modeling. Spots for which the $\log _{2} \mathrm{SD}$ of the triplicates was more than 0.2 were discarded.

\section{Mutation detection}

$B R A F$ codon 600 and NRAS codon 61 were screened for mutations on a LightCycler real-time PCR System (Roche Diagnostics GmbH, Mannheim, Germany) by melting curve analysis using fluorescent probes, as we have previously described [11]. The accuracy of this method was confirmed by direct sequencing of PCR products that showed deviation from the WT genomic DNA melting peak. 
Table 1 Clinical and histopathological parameters of patients with primary melanomas

\begin{tabular}{lc}
\hline Variables & Number of primary melanomas \\
\hline All tumors & $47(100 \%)$ \\
Tumor type & $19(40.4 \%)$ \\
NM & $28(59.6 \%)$ \\
SSM & \\
Sex & $24(51.1 \%)$ \\
Male & $23(49 \%)$ \\
Female & \\
Age (years) & $15(31.9 \%)$ \\
20-50 & $32(68.1 \%)$ \\
>50 & \\
Breslow thickness $(\mathrm{mm})^{\mathrm{a}}$ & $17(36.2 \%)$ \\
<2.01 & $9(19.1 \%)$ \\
$2.01-4.00$ & $21(44.7 \%)$ \\
>4.00 & $20(42.6 \%)$ \\
Clark's level & $27(57.4 \%)$ \\
I, II, III & \\
IV, V & $19(40.4 \%)$ \\
Ulceration & $28(59.6 \%)$ \\
Absent & \\
Present & $20(42.6 \%)$ \\
Metastasis formation & $27(57.4 \%)$ \\
Nonmetastatic & \\
Metastatic & $29(61.8 \%)$ \\
Patient survival & $18(38.3 \%)$ \\
Alive & \\
Exitus & $26(57.8 \%)$ \\
BRAF & $19(40.4 \%)$ \\
Absent & $2(4.3 \%)$ \\
Present & $37(84.1 \%)$ \\
NA & $7(15.9 \%)$ \\
NRAS & $3(6.4 \%)$ \\
Absent c & \\
Present & \\
NA & \\
\hline N & \\
\hline
\end{tabular}

NM, nodular melanoma; SSM, superficial spreading melanoma.

${ }^{a}$ Thickness categories on the basis of the current melanoma staging system.

bThe distribution of the BRAF codon 600 mutation.

'The distribution of the NRAS codon 61 mutation.

\section{Real-time quantitative PCR analysis}

Real-time quantitative PCR was used to evaluate the differential fold changes between the target and the reference genes in 17 primary melanoma samples and to confirm the aCGH results. The DNA copy-number alterations of eight BAC clones localized on the $11 \mathrm{q} 13.3$ region (CTD-2192B11, CTB-36F16, RP1-88B16, RP1162F2, RP1-4E16, RP1-128I8, RP1-17L4, and CTC437H15; start bp: 69070147, end bp: 69960225) were validated by quantifying the relative amounts of four target genes (CCND1, FGF3, FGF4, and FGF19), all located in this particular chromosomal region, with real-time quantitative PCR using two reference genes for normalization, GNS (12q14.3) and UBE2E1 (3p24.2), as we previously described [11].

\section{Fluorescence in situ hybridization}

Dual-color fluorescence in situ hybridization (FISH) probe (Vysis, Downers Grove, Illinois, USA) was used on selected tumor samples to detect copy number alterations on amplified and deleted regions, including DNA probes specific for 9p21, 7q31, as described previously [16,17].

\section{Data analysis}

All BAC clones were mapped to the human genome (February 2009) using data provided by the UCSC genome browser site (http://genome.ucsc.edu/). From data processing, all X-chromosome and Y-chromosome clones were excluded. BAC clones, which are known to have genomic variants according to the Database of Genomic Variants (The Centre for Applied Genetics, Toronto, Ontario, Canada, http://projects.tcag.ca/variation/), were omitted. The $\log _{2}$-transformed data were subjected to copynumber change analyses for the identification of regions of amplification and deletion. To determine gains and losses of each regions, the Analysis of Copy Errors algorithm in CGH Explorer software 3.2 (Department of Informatics, The Faculty of Mathematics and Natural Sciences, Blindern, Oslo, Norway) was used with a false discovery rate (FDR) of less than 0.01 [18]. Previously, 11 different algorithms were compared, which are most frequently used for analyzing aCGH data [19]. In this paper, they pointed out that some current implementations do not include any assessment of the statistical significance of the reported copy number changes, although quantitative statistics of the aberrations are critical to decide which region to pursue for further analysis. Analysis of Copy Errors is one of the two algorithms that incorporates FDR so far. The CGH Explorer was also used to obtain graphical illustrations of copy number alteration frequencies in primary melanomas.

For the subsequent identification of high-level gains and homozygous deletions in aCGH data, ratio thresholds were used as described in previous studies [8]. These were more than 0.55 ( $>\sim 5$ copies) and less than -0.8 , respectively. Estimates of genome-wide aberration rates were carried out by simply calculating the proportion of $\mathrm{BAC}$ clones gained or lost in a specific tumor sample.

To identify BAC clones or regions that differ in copy number between tumor subgroups, Fisher's exact test was applied. We used an FDR correction procedure to adjust for multiple comparisons and denote these resulting $P$ values as adjusted $P$ values [20]. To increase our power for identifying regional changes in copy number between tumor subgroups, we averaged $\log _{2}$ ratios over windows of five consecutive BAC clones and used a twosample $t$-statistic to compare the average $\log _{2}$ ratio for the tumor subgroups for each window. We calculated an adjusted $P$ value using a permutation-based procedure of Westfall and Young [21].

Identification of the correlations between BAC clones or regions in different tumor subgroups $\left(\mathrm{BRAF}^{\mathrm{mut}}, \mathrm{WT}\right)$ was achieved by calculating a standard Pearson's correlation, which defines the magnitude and direction of the linear relationship between BAC clones to quantify whether these are changing in a concordant, discordant, or unrelated manner. First, a subset of BAC clones was chosen to distinguish the subgroups (see the following for 
specific criteria) and then a pairwise correlation was computed for all of the BAC clones from the subset. BAC clones were selected if either the difference in the BAC's gain or loss percentages between the tumor subgroups (BRAF $^{\text {mut }}$, NRAS ${ }^{\text {mut }}$ and WT) was more than $40 \%$ or if its adjusted $P$ value from the categorical or the windowed analysis was less than 0.3 (similar to that applied by Loo et al. [22]). We have chosen these selection criteria to include BAC clones that may truly differ between tumor subgroups but did not achieve statistical significance because of insufficient power. It should be emphasized that this procedure of selecting BAC clones does not bias the correlation analysis because these selection criteria were based on comparison between tumor subgroups, whereas the correlations were calculated within each subgroup. All the cited $P$ values were adjusted for multiple comparisons. The analyses were carried out in the opensource statistical computing environment R (http://www.rproject.org/).

\section{Investigating the frequency of cross-talks changed in primary melanomas}

Cancers are often viewed as systems diseases [23]. In cancer cells, large-scale modifications of signaling pathways, especially in cross-talks, are prevalent [24]. The definition of cross-talk is as follows: if two proteins belong to different pathways, then the signaling interaction between these two proteins is a cross-talk between their pathways. To identify cross-talks, we used the SignaLink database (http://signalink.org/), which provides a precise mapping of signaling pathways and also inform if a signaling protein belongs to more than one pathway [25]. To assess gene alterations in eight gene signaling networks [EGF/MAPK kinase (EGF), Insulin/IGF (IGF), TGF- $\beta$ (TGF), Wingless/WNT (WNT), Hedgehog (HH), JAK/ STAT (JAK), Notch (Notch), and Nuclear Hormone Receptor (NHR)] we estimated the copy number changes of each of these pathway genes using the closest BAC clone within $2 \mathrm{Mb}$. We considered a signaling interaction to be altered if the copy-number change of at least one of the participating genes was classified as a gain or a loss by aCGH analysis, and then we simply calculated the average frequency of altered cross-talks within and between different pathways for one particular tumor subgroup.

\section{Random forest analysis}

The random forest package [26,27] of the R-statistical programming language (http://www.r-project.org/) was applied to calculate the random forest classification and importance measures on the aCGH data related to the $\mathrm{EGF} / \mathrm{MAPK}$ pathway genes (clone number $=138$ ). The feature importance score derived from the random forest classifier was used to assess the association of a particular set of genes with positive $B R A F$ mutations. The parameters were set as follows: ntree $=5000$ (number of trees) and mtry $=11$ (the number of randomly selected variables per branching of the tree). The most important
15 genes were listed and sorted by their importance measures (mean decrease Gini and mean decrease accuracy) over 1000 simulation runs and an automatic rerun was performed with a value of 3 for the mtry parameter using only those 15 variables that were most important in the original run. Cross-validation was performed; thus, the model was developed on the training set $(60 \%)$ and validated on the test set (40\%) of tumors.

\section{Results \\ Mutation frequencies of BRAF and NRAS oncogenes in primary melanomas}

The mutation status for BRAF and NRAS oncogenes $\left(\mathrm{BRAF}^{\mathrm{mut}}\right.$ or $\mathrm{BRAF}^{\mathrm{WT}}$, NRAS ${ }^{\text {mut }}$ or NRAS $\left.{ }^{\mathrm{WT}}\right)$ was successfully defined for 44 tumors. Fifty-nine percent (26/44) of primary melanomas had either $B R A F$ or $N R A S$ mutations, but both mutations were never simultaneously present in any of the samples analyzed. $B R A F$ mutations at codon 600 were found in $40 \%$ of lesions. NRAS mutations at codon 61 were detected in only $16 \%$ of tumors (Table 1). The BRAF mutation was significantly associated with tumor thickness, being more frequent in samples with more than $2 \mathrm{~mm}$ Breslow thickness. The NRAS mutation was significantly associated with metastasis formation (primary data summarized in Table 2).

\section{Genomic alterations in $\mathbf{4 7}$ primary cutaneous melanoma cancer samples}

We analyzed tumor DNA from 47 frozen tissue primary melanoma samples. To identify the overall trends across all of the tumors, we plotted the frequency of tumors showing gain or loss for each BAC clones across the genome (Fig. 1). We have listed the high frequency $(>30 \%$ of tumors) of regional gains $(>5 \mathrm{Mb})$ and losses in Table 3 .

\section{Correlation of copy number alterations with BRAF or NRAS mutation status}

To identify genomic alterations associated with $B R A F$ or $N R A S$ mutational status, we compared three groups of tumors: (a) BRAF $^{\text {mut }}$ primary melanoma $(n=19)$; (b) NRAS $^{\text {mut }}$ primary melanoma $(n=7)$; and (c) WT (wildtype for both loci) primary melanoma $(n=18)$.

First, we compared the gain and loss frequencies in mutation groups $\left(\mathrm{BRAF}^{\mathrm{mut}}, \mathrm{NRAS}^{\mathrm{mut}}, \mathrm{WT}\right)$. The average frequency of copy number changes was higher in BRAF $^{\text {mut }}$ tumors than in WT tissues (Mann-Whitney test; $P=0.04$ and 0.01 , for gains and losses, respectively).

Table 2 Associations of BRAF and NRAS mutations with patients' clinicopathological parameters

\begin{tabular}{lcc}
\hline & Breslow thickness $>2 \mathrm{~mm}$ & $P$ value \\
\hline $\operatorname{BRAF}^{\text {mut }}(n=19)$ & 17 & 0.009 \\
$\operatorname{BRAF}^{\mathrm{WT}}(n=26)$ & 13 & \\
NRAS $^{\text {mut }}(n=7)$ & Metastasis formation & \\
NRAS $^{\text {WT }}(n=37)$ & 7 & 0.031 \\
\hline
\end{tabular}


Fig. 1

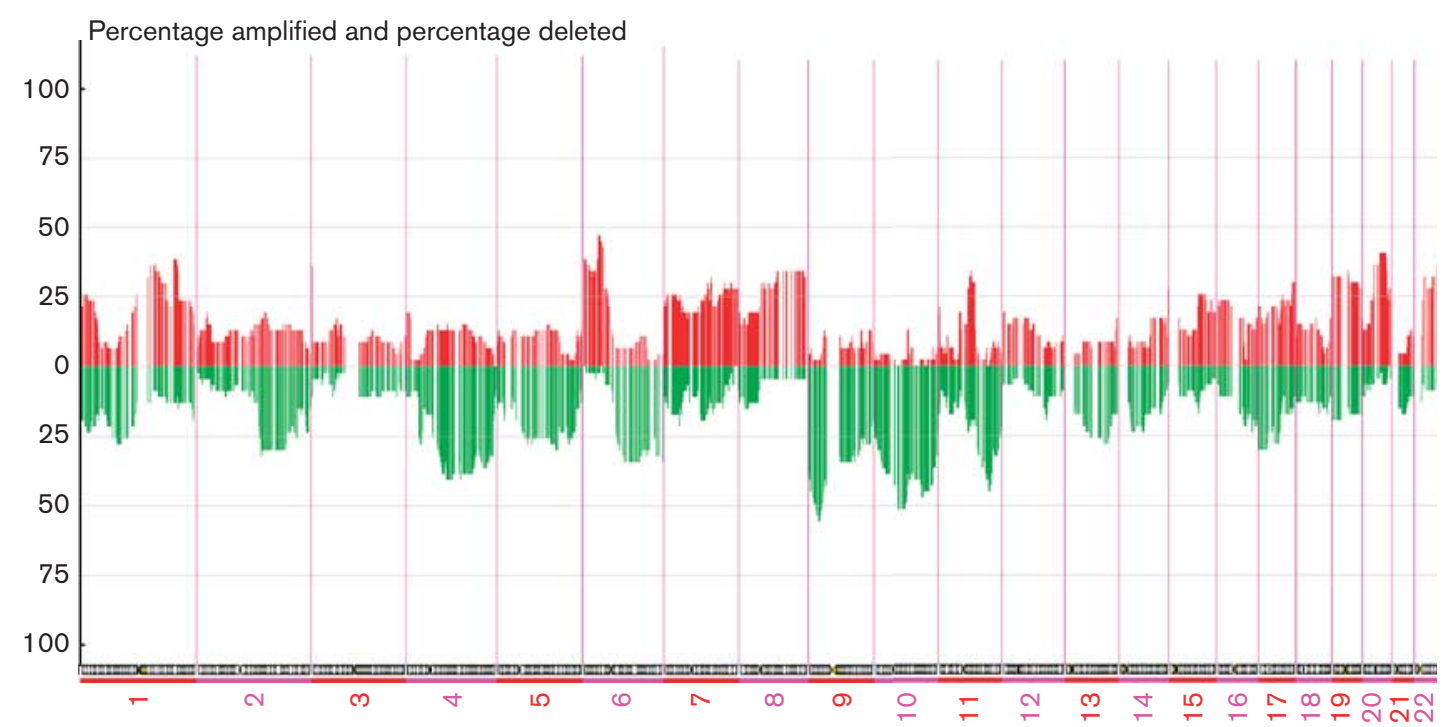

Overall frequency of BAC copy number gain and loss for 47 primary melanomas. The percentage of the 47 tumors showing gain (red; above 0 ) or loss (green; below 0) of DNA represented by each of the 2379 BAC is plotted against the corresponding genomic position of the BAC clone. BAC, bacterial artificial chromosome.

However, no other differences were found in genomewide aberration rates between these groups.

The frequency patterns of copy number changes for each group across the entire genome are shown in Fig. 2. We used four different methods to identify BAC clones or groups of BAC clones that showed more frequent loss or gain in one tumor subgroup than another: (a) measurements of difference in the frequency of gain or loss between subgroups were higher than 40\%; (b) determination of significant differences in copy number changes in individual BAC clones between subgroups using Fisher's exact test; (c) determination of statistical difference between windows of five BAC clones with a two-sample $t$-statistic; and (d) characterization of high-level loss and gain in more than $20 \%$ of tumors in each subtype.

We have listed distinct chromosomal regions in Table 4, which shows more frequent gains and losses $(>40 \%)$ in one tumor subgroup than in another. Alterations exclusively associated with $\mathrm{BRAF}^{\mathrm{mut}}$ were the gain of chromosome 7 and 1q23-q25 and losses on the long arm of chromosome 10. Losses of the 6q25.3-6q27, 11q23.3$\mathrm{q} 25$, and $17 \mathrm{p} 13.3$ loci were the most common DNA alterations in NRAS ${ }^{\text {mut }}$ tumor samples. Loss of the 9p21.3 region and gain of $8 \mathrm{q}$ were more frequent in melanomas with BRAF $^{\text {mut }}$ or NRAS ${ }^{\text {mut }}$. Primary melanoma without $B R A F$ or $N R A S$ mutations was primarily characterized by alterations in chromosomes 17 and 4.

Using Fisher's exact test to compare gain and loss of individual $\mathrm{BAC}$ clones between $\mathrm{BRAF}^{\text {mut }}$ and $\mathrm{WT}$ tumor subgroups, we found that the loss of 45 clones from
Table 3 High frequency $(>30 \%)$ of regional $(>5 \mathrm{Mb})$ gains and losses in 47 primary melanomas

\begin{tabular}{llr}
\hline Chromosome location & Event & Number of BAC clones \\
\hline $1 p 36.31-p 36.21$ & Gain & 7 \\
$1 q 21.1-1 q 25.3$ & Gain & 24 \\
$1 q 31.3-1 q 32.1$ & Gain & 11 \\
$6 p 25.3-6 p 12.3$ & Gain & 43 \\
$7 q 31.2-7 q 31.33$ & Gain & 11 \\
$8 q 11.21-8 q 12.3$ & Gain & 20 \\
$8 q 21.11-8 q 24.3$ & Gain & 57 \\
$11 q 13.1-11 q 13.4$ & Gain & 29 \\
$15 q 22.2-15 q 25.1$ & Gain & 20 \\
$17 q 25.1-17 q 25.3$ & Gain & 17 \\
$19 p 13.3-19 q 13.42$ & Gain & 36 \\
$20 p 11.21-20 q 13.2$ & Gain & 44 \\
$22 q 11.21-22 q 13.32$ & Gain & 16 \\
$1 p 36.22-1 p 35.2$ & Loss & 15 \\
$2 q 22.1-2 q 32.2$ & Loss & 49 \\
$4 q 13.3-q 35.1$ & Loss & 107 \\
$5 q 22.3-5 q 23.2$ & Loss & 8 \\
$6 q 13-6 q 27$ & Loss & 37 \\
$9 p 24.3-9 q 32$ & Loss & 102 \\
$10 p 15.3-10 q 26.3$ & Loss & 51 \\
$11 q 14.1-11 q 24.2$ & Loss & 20 \\
$13 q 14.3-13 q 31.3$ & Loss & 46 \\
$17 p 13.3-17 q 21.32$ & Loss & \\
\hline 19 & &
\end{tabular}

BAC, bacterial artificial chromosome.

the 10q23.3-10q26.3 region was mainly associated with BRAF $^{\text {mut }}$ melanoma (adjusted $P$ values $<0.05$ ). Using a slightly less conservative level of significance (adjusted $P<0.1$ ), we identified an additional 73 BAC clones at the following locations: gains in $7 \mathrm{p} 14.2-7 \mathrm{q} 11.22$ and $7 \mathrm{q} 36.3$ and losses in $1 \mathrm{p} 33$ and $10 \mathrm{q} 21.1-10 \mathrm{q} 23.31$, which were found to be more frequent in $\mathrm{BRAF}^{\text {mut }}$ than in WT tumors. Moreover, 29 BAC clones exhibited differences between NRAS ${ }^{\text {mut }}$ and WT tumor subgroups (adjusted 
Fig. 2
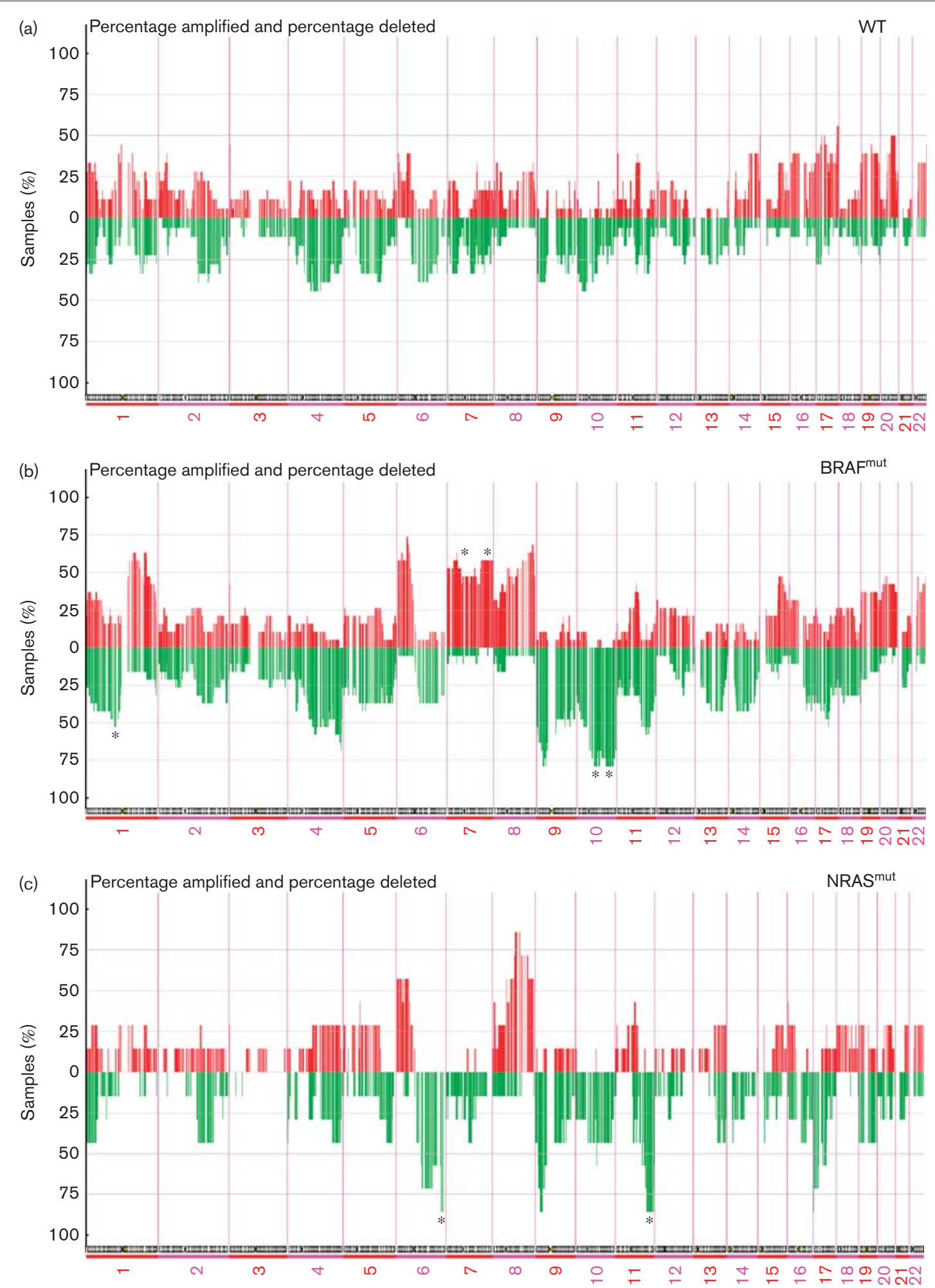

Frequency plots of copy number gain and loss in subgroups of primary melanomas. The percentages of copy number gain (red; above 0 ) and loss (green; below 0) were calculated for (a) WT $(n=18)$, (b) BRAF ${ }^{\text {mut }}(n=19)$, and (c) NRAS ${ }^{\text {mut }}(n=7)$ tumor tissues. *Regions significantly altered (adjusted $P$ value $<0.05$ ) (or close to; adjusted $P$ value $<0.1$ ) between mutation subgroups. WT, wild-type. 
Table 4 Chromosomal loci that showed more frequent (more than $40 \%$ ) gain or loss in one tumor subtype than another (BRAF ${ }^{m u t}$, NRAS $^{\text {mut }}$, WT)

\begin{tabular}{|c|c|c|c|}
\hline Chromosome location & Event & Number of BAC clones ${ }^{a}$ & Association \\
\hline \multicolumn{4}{|l|}{ BRAF $^{\text {mut }}$ vs. NRAS ${ }^{\text {mut }}$} \\
\hline $1 q 23.2-1 q 25.2$ & Gain & 16 & BRAF $^{\text {mut }}$ \\
\hline $7 \mathrm{chr}$ & Gain & 191 & BRAF $^{\text {mut }}$ \\
\hline $20 q 13.32$ & Gain & 1 & BRAF $^{\text {mut }}$ \\
\hline 1p34.2-1p33 & Loss & 3 & BRAF $^{\text {mut }}$ \\
\hline $1 \mathrm{p} 13.2$ & Loss & 5 & BRAF $^{\text {mut }}$ \\
\hline $4 q 13.3$ & Loss & 3 & BRAF $^{\text {mut }}$ \\
\hline $4 q 22.1-4 q 25$ & Loss & 3 & BRAF $^{\text {mut }}$ \\
\hline $10 q 21.3-10 q 22.1$ & Loss & 2 & BRAF $^{\text {mut }}$ \\
\hline $10 q 26.13-10 q 26.3$ & Loss & 15 & BRAF $^{\text {mut }}$ \\
\hline $6 q 25.3-6 q 27$ & Loss & 4 & NRAS ${ }^{\text {mut }}$ \\
\hline $11 q 23.3-11 q 25$ & Loss & 21 & NRAS ${ }^{\text {mut }}$ \\
\hline $17 p 13.3$ & Loss & 2 & NRAS ${ }^{\text {mut }}$ \\
\hline \multicolumn{4}{|l|}{ BRAF $^{\text {mut }}$ vs. WT } \\
\hline $1 q 24.1-q 24.3$ & Gain & 4 & BRAF $^{\text {mut }}$ \\
\hline $1 q 25.3-1 q 31.2$ & Gain & 4 & BRAF $^{\text {mut }}$ \\
\hline $6 p 22.3$ & Gain & 7 & BRAF $^{\text {mut }}$ \\
\hline $7 p 22$ & Gain & 11 & BRAF $^{\text {mut }}$ \\
\hline $7 p 21.3-7 p 21.1$ & Gain & 3 & BRAF $^{\text {mut }}$ \\
\hline $7 p 15.3-7 q 36.3$ & Gain & 128 & BRAF $^{\text {mut }}$ \\
\hline $8 q 11.11-8 q 11.22$ & Gain & 4 & BRAF $^{\text {mut }}$ \\
\hline $8 q 24.11-8 q 24.3$ & Gain & 31 & BRAF $^{\text {mut }}$ \\
\hline $1 p 33-1 p 32.3$ & Loss & 3 & BRAF $^{\text {mut }}$ \\
\hline $9 p 21.1-9 p 13.3$ & Loss & 6 & BRAF $^{\text {mut }}$ \\
\hline $9 p 13.2$ & Loss & 1 & BRAF $^{\text {mut }}$ \\
\hline $10 q 11.21-10 q 26.3$ & Loss & 93 & BRAF $^{\text {mut }}$ \\
\hline $11 q 22.1$ & Loss & 1 & BRAF $^{\text {mut }}$ \\
\hline $11 q 23.1-11 q 23.2$ & Loss & 6 & BRAF $^{\text {mut }}$ \\
\hline 11q14.2-11q23.3 & Loss & 15 & $\mathrm{BRAF}^{\mathrm{mut}}$ \\
\hline \multicolumn{4}{|l|}{ NRAS $^{\text {mut }}$ vs. WT } \\
\hline $8 q 12.2-8 q 21.11$ & Gain & 14 & NRAS $^{\text {mut }}$ \\
\hline $8 q 24.23-8 q 24.3$ & Gain & 3 & NRAS ${ }^{\text {mut }}$ \\
\hline $6 q 22.31$ & Loss & 2 & NRAS $^{\text {mut }}$ \\
\hline $6 q 25.2-6 q 27$ & Loss & 6 & NRAS $^{\text {mut }}$ \\
\hline $9 p 22.2-9 p 21.3$ & Loss & 5 & NRAS $^{\text {mut }}$ \\
\hline $11 q 21-11 q 25$ & Loss & 38 & NRAS ${ }^{\text {mut }}$ \\
\hline $17 p 13.3-17 p 11.2$ & Loss & 30 & NRAS $^{\text {mut }}$ \\
\hline $17 q 12-17 q 21.2$ & Loss & 15 & NRAS $^{\text {mut }}$ \\
\hline $4 q 23-4 q 25$ & Loss & 4 & WT \\
\hline $17 p 11.2$ & Gain & 4 & WT \\
\hline $17 q 24.3-17 q 25.3$ & Gain & 15 & WT \\
\hline
\end{tabular}

BAC, bacterial artificial chromosome.

${ }^{a}$ Number of BAC clones altered in the region.

$P$ value $<0.1)$. Loss in $6 \mathrm{q} 25.2-6 \mathrm{q} 25.3$ and $11 \mathrm{q} 23.1-11 \mathrm{q} 25$ was mainly seen in tumors with NRAS ${ }^{\text {mut }}$, but none of these alterations were commonly seen in WT tumors. Asterisks on Fig. 2 indicate the location of the BAC clones showing differences between these primary melanoma subgroups with this test. Our data show that the BAC clones achieving a level of significance with Fisher's exact test are often part of a larger region that differs in the frequency of gain or loss between these tumor subtypes.

To increase our power in identifying copy number gains and losses larger than those identified by single BAC clones, we applied a two-sample $t$-statistic comparing a sliding window of five consecutive BAC clones in the tumor subgroups. With this test, we observed differences between NRAS ${ }^{\text {mut }}$ and WT tumor subgroups for BACs at the following locations: 11q23.2, 11q24.1-11q24.2, and $11 \mathrm{q} 25$ (adjusted $P$ values $<0.1$ ). There was also a suggestive evidence of differences between $\mathrm{BRAF}^{\text {mut }}$ and WT tumor subgroups in 7q11.22, 7q11.23, 7q21.11, 10q11.23, 10q23.1, 10q23.1, 10q23.33-10q24.33, and 14q24.3 (adjusted $P$ values $<0.1$ ).

To further characterize genomic differences between tumor subgroups, we also compared the frequency with which particular BAC clones showed high-level amplifications and homozygous deletions exceeding the upper or lower thresholds $\left(\log _{2}\right.$ ratio $\geq 0.55$ or $\leq-0.8$, respectively). BAC clones showing such alterations in at least $20 \%$ of the tumors in a subgroup are listed in Table 5. Frequent homozygous deletion was seen in both $\mathrm{BRAF}^{\text {mut }}$ and WT melanomas in the $17 \mathrm{q} 21.32$ region harboring the HOXB3-9 gene cluster, which are members associated with many malignant tumors [28]. Homozygous deletion of the CDKN2A (9p21.3) gene was found only in $8.5 \%$ of tumors $(4 / 47)$.

\section{Correlation of gain or loss changes in BRAF ${ }^{\text {mut }}$ and wild-type primary melanomas}

Pairwise correlation analysis of the subset of $\mathrm{BRAF}^{\mathrm{mut}}$ and WT tumors (including 519 and 167 BAC clones, respectively; these clones exhibited more frequent loss or gain in these tumor subgroups) revealed chromosomal alterations that coexist more often together in these groups of tumors. The heat maps of Fig. 3 show regions of positive (change in same direction: green) and negative (change in opposite direction: red) correlations between certain regions. There were positive correlations (correlation coefficient $>0.7$ ) between (a) loss in 1p34.2-1p32.2 and loss in 4q22.1-4q25; (b) loss in 1p13.2 and loss in 4q22.1-4q24; (c) loss in 1p21.3-p13.2 and loss in 14q23.2; (d) loss in 4q35.1 and loss in 11q23.2-q23.3; and (e) gain in $7 \mathrm{q} 21.11-7 \mathrm{q} 31.1$ and gain in 20p12.2-20p12.1 in the $\mathrm{BRAF}^{\text {mut }}$ tumor group. Relatively large regions of negative correlation (correlation coefficient $<-0.7$ ) were seen in WT tumors between changes in the copy number of BAC clones such as: (a) loss in 4q13.1-4q13.3 and gain in 14q24.1-14q32.2; (b) loss in 4q13.1-4q13.3 and gain in $17 \mathrm{q} 24.3-17 \mathrm{q} 25.3$; (c) loss in $4 \mathrm{q} 23-4 \mathrm{q} 25$ and gain in 14q24.1-14q32.2; (d) loss in $4 \mathrm{q} 25$ and gain in 17q24.3-17q25.3; (e) loss in 7q11.23-7q21.11 and gain in $17 \mathrm{p}$; (f) loss in $7 \mathrm{q} 31.1-17 \mathrm{q} 31.2$ and gain in $17 \mathrm{p}$; and (g) loss in 7q31.31-7q31.32 and gain in 7p. Furthermore, gain of several BAC clones from 14q24.1-14q32.2 showed a positive correlation (correlation coefficient $>0.7$ ) with copy number gain in the $17 \mathrm{q} 25.1-\mathrm{q} 25.3$ region in primary melanomas without the BRAF or the NRAS mutation.

\section{Identification of gene signature associated with the BRAF mutation in the EGF/MAPK pathway}

Because the $B R A F$ oncogene is one of the key activators of the EGF/MAPK pathway, we performed a focused analysis of this signaling pathway to investigate whether there are any gene signatures in this pathway that are related to the $B R A F$ mutation. We estimated the copy number changes of each of these pathway genes using the closest $\mathrm{BAC}$ clone within $2 \mathrm{Mb}$. Using the random forest 
Table 5 The list of regions where the most common high-level amplification and homozygous deletions were observed

\begin{tabular}{lll}
\hline Chromosome location & \multicolumn{1}{c}{ Event } & \multicolumn{1}{c}{ Association $^{\text {a }}$} \\
\hline $6 p 22.3$ & High-level amplification & BRAF $^{\text {mut }}$ \\
$7 p 21.3$ & High-level amplification & BRAF $^{\text {mut }}$ \\
$7 p 21.1$ & High-level amplification & BRAF $^{\text {mut }}$ \\
$7 p 15.3$ & High-level amplification & BRAF $^{\text {ut }}$ \\
$7 p 14.3$ & High-level amplification & BRAF $^{\text {mut }}$ \\
$7 p 14.1$ & High-level amplification & BRAF $^{\text {mut }}$ \\
$7 p 13$ & High-level amplification & BRAF $^{\text {mut }}$ \\
$7 p 12.3$ & High-level amplification & BRAF $^{\text {ut }}$ \\
$7 p 12.1$ & High-level amplification & BRAF $^{\text {mut }}$ \\
$7 q 31.2$ & High-level amplification & BRAF $^{\text {mut }}$ \\
$7 q 32.2$ & High-level amplification & BRAF $^{\text {mut }}$ \\
$7 q 35-q 36.3$ & High-level amplification & BRAF $^{\text {ut }}$ \\
$8 q 24.13$ & High-level amplification & BRAF $^{\text {mut }}$ \\
$17 q 21.32$ & Homozygous deletion & BRAF $^{\text {mut }}$ \\
6p22.3 & High-level amplification & NRAS $^{\text {mut }}$ \\
$6 p 21.31$ & High-level amplification & NRAS $^{\text {mut }}$ \\
$6 p 12.1$ & High-level amplification & NRAS $^{\text {mut }}$ \\
$13 q 21.33-13 q 22.1$ & High-level amplification & NRAS $^{\text {mut }}$ \\
$13 q 31.3-13 q 32.1$ & High-level amplification & NRAS $^{\text {mut }}$ \\
$13 q 33.1$ & High-level amplification & NRAS $^{\text {mut }}$ \\
$13 q 33.3$ & High-level amplification & NRAS $^{\text {mut }}$ \\
& &
\end{tabular}

alt was considered to be associated with a tumor subtype if at least $20 \%$ of primary melanomas in that particular subgroup showed this genetic alteration.

classifier among the EGF/MAPK pathway genes, we identified a signature of 15 genes that was highly predictive for a $B R A F$-positive mutation status. A cross-validation test was carried out for each set of genes to evaluate the accuracy measures of the random forest model: the overall accuracy was $87.5 \%$ (number of correct classifications), sensitivity was $77.8 \%$ (the proportion of true positives), and specificity was $70 \%$ (the proportion of true negatives). Frequent coamplification of well-known oncogenes such as $E G F R, P I 3 K$, and several less known genes, such as SHC1, PEA15, ELK4, was also found to be most likely associated with the presence of the $B R A F$ mutation. We also observed a common deletion pattern in three well-known tumor suppressor genes, PTEN, JNK1, and $H V H-3$, which fell into these predictive sets of genes and several less known genes, such as JNKK2, MEK2, ATF2, TPBG, SOS1, SHOC2, and TGFBR1.

\section{Frequent changes in signaling cross-talks in primary melanomas harboring the BRAF mutation}

We also aimed to investigate the frequency of copy number changes of the signaling interactions between and within eight tumor regulatory pathways in primary melanoma subgroups with different $B R A F$ genotypes $\left(\mathrm{BRAF}^{\text {mut}}\right.$ : tumors harboring the BRAF mutation; $\mathrm{BRAF}^{\mathrm{WT}}$ : tumors without the $B R A F$ mutation, which also includes tumors with the $N R A S$ mutation). The results of this analysis are shown in Fig. 4. We observed that cross-talk between the EGF and the JAK networks is extensively altered in the BRAF ${ }^{\text {mut }}$ tumors compared with the BRAF ${ }^{\mathrm{WT}}$ lesions. In addition, interactions within the EGF-JAK, JAK-IGF, and EGF-IGF pathways are more frequently altered $(>20 \%)$. This analysis further supported the potentially important role of the $\mathrm{HH}$ pathway in $\mathrm{BRAF}^{\text {mut }}$ primary melanoma.
Validation of array comparative genomic hybridization data by Q-PCR and fluorescence in situ hybridization

To validate some frequently found $(>30 \%$ of primary melanomas) regional gains in $11 \mathrm{q} 13,7 \mathrm{q} 31$, and losses in 9p21, we performed a detailed Reverse transcription polymerase chain reaction and FISH analysis. Real-time quantitative PCR for four target genes located within the 11q13.3 chromosomal segment was performed. A good concordance was found between data derived from QPCR and aCGH for 17 different samples. A mathematical comparison was also conducted between the two experimental approaches. Spearman's rank correlation was carried out using the mean $\log _{2}$ values of each BAC clone covering this particular region of interest, and the mean $\log _{2}$ ratio of each target gene to reference genes was revealed by Q-PCR. The correlation coefficient showed strong correlations between the two results (Spearman's rank correlation $\rho=0.7, P=0.003$ ).

FISH was also applied for the following regions: 7q31, 9p21 for selected samples. Good agreement was found between the two methods. An average of 5.8 copies (range of 2.5-13.3) of 7q31 signals was found among the 10 cases that exhibited gain by aCGH analysis. All the tumors that exhibited 7q31 gain by aCGH also showed gain by FISH. Furthermore, an average of 1.6 copies (range: 2.8-0.2) of $9 \mathrm{p} 21$ signals was detected among the 17 cases that exhibited loss by aCGH analysis. In four samples, loss of the 9p21 region was detected by aCGH; however, the average copy number of the $9 \mathrm{p} 21$ signal was $2.1,2.1,2.7$, and 2.8 by FISH. The reason for this discrepancy may be that the touch preparation and the DNA were from slightly different parts of the tumor or a high copy number heterogeneity existed within the sample.

\section{Discussion}

In this study, we used high-resolution array CGH to evaluate the copy number changes in primary cutaneous melanomas. aCGH identifies genomic imbalance at a level of resolution higher than that achievable by classical cytogenetic analysis. The higher resolution of genomic screening has allowed a more detailed evaluation of the DNA content. On the basis of the analysis of the 47 primary melanomas, we observed large regional gains in 1p36, 1q, 6p, 7q31, 8q, 11q13, 15q, 17q25, chromosome $20,22 \mathrm{q}$ and losses in $2 \mathrm{q}, 4 \mathrm{q}, 5 \mathrm{q} 22-\mathrm{q} 23,6 \mathrm{q}, 9$, chromosome 10 and $17,11 \mathrm{q} 14-\mathrm{q} 24$, and $13 \mathrm{q}$, many of which have been reported by previous studies using conventional cytogenetic and CGH methods [29-31].

Through DNA copy-number profiling, we aimed to discern differentially altered chromosomal segments and genes between $B R A F$ and $N R A S$ mutated melanoma subgroups. Somatic mutations of $B R A F$ and $N R A S$ oncogenes are thought to be among the first steps in melanoma initiation and it has been proved that they are preserved throughout tumor progression. Therefore, drugs targeting 
Fig. 3

(a)

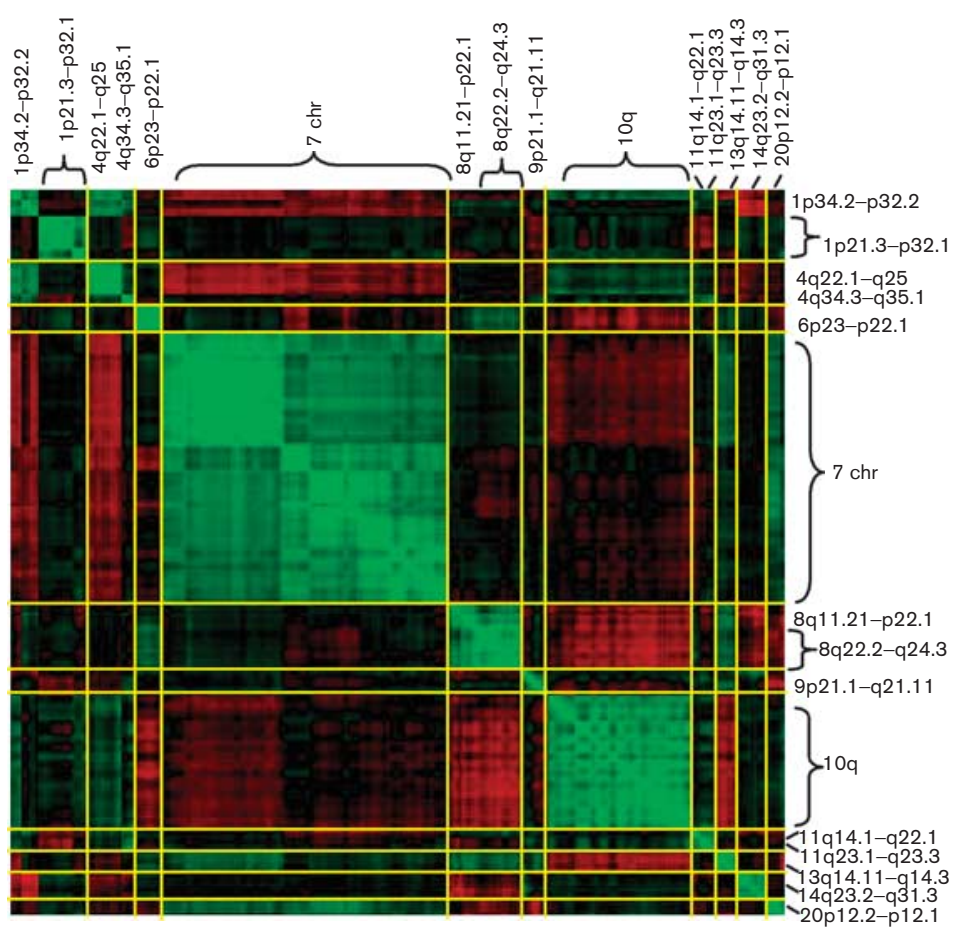

(b)

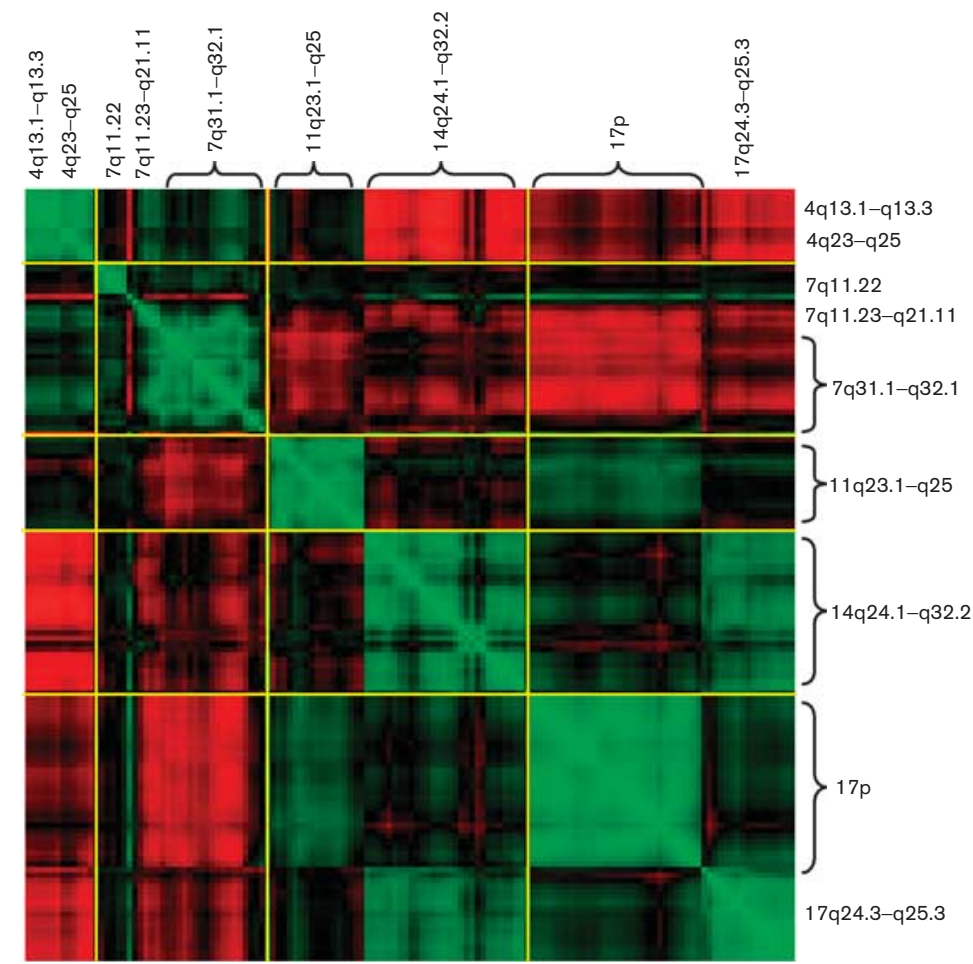

Correlation matrix of copy number changes for $\mathrm{BRAF}^{\mathrm{mut}}$ and WT primary melanomas. The heat maps show a positive (change in same direction; green) and a negative (change in opposite direction; red) correlation between loss or gain of individual BAC clones for the 19 BRAF ${ }^{\text {mut }}$ (a) and 18 WT (b) tumors. The 519 or 167 BACs clones that showed a frequent change in BRAF $^{\text {mut }}$ or WT tumors are shown in genome order. Chromosomal band information is labeled. BAC, bacterial artificial chromosome; WT, wild-type. 
Fig. 4

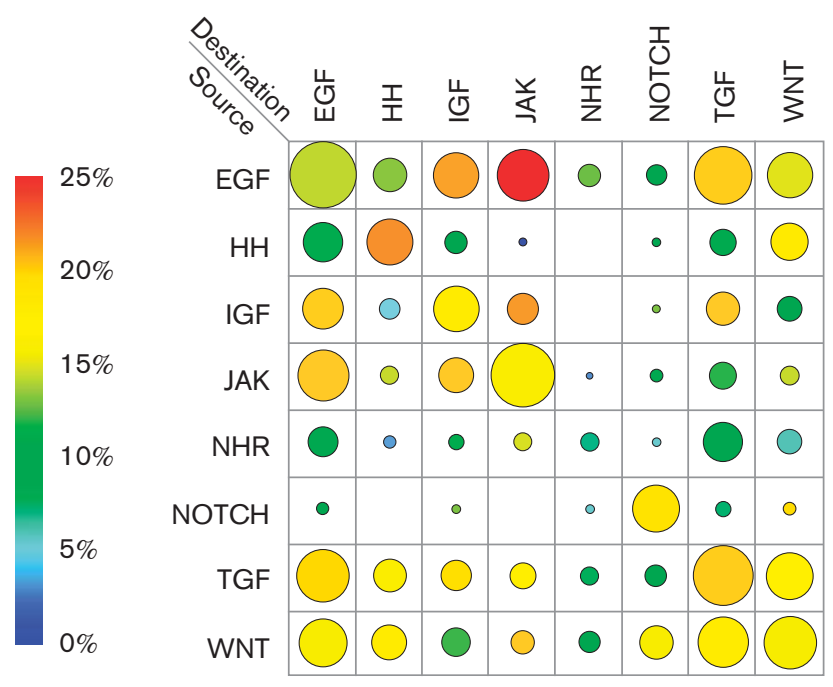

Differences in the frequency of altered cross-talks between BRAFmut and BRAF ${ }^{\mathrm{WT}}$ primary melanomas. The number of cross-talks is indicated by varying circle sizes. The lack of a circle indicates that there are no cross-talks between the pathways. We calculated the differences in the frequency of altered cross-talks by subtracting the average frequency in BRAFWT tumor subtype from the average frequency in BRAF ${ }^{\text {mut }}$; the results are displayed as a heat map. The signal flow direction in the signaling interaction network is labeled as 'source' or 'destination' on the figure (i.e. a protein from the source pathway activates or inhibits a protein from the destination pathway). Broad ranges of signaling interactions were more frequently altered $(>20 \%)$ in BRAF ${ }^{\text {mut }}$ melanoma compared with the BRAFWT tumor type within the $\mathrm{HH}$ and between the EGF-JAK, IGF-JAK, and EGF-IGF pathways.

this pathway are the most attractive clinical agents for the disease $[32,33]$. However, characterization of gene copy number anomalies in primary melanomas in conjunction with $B R A F$ and $N R A S$ mutational status can provide rational additional targets for a combination therapy, which may help to enhance the response and decrease the resistance to $B R A F$ and NRAS inhibitors.

Previous studies have revealed distinct genetic alterations between melanoma subgroups on the basis of mutations of the BRAF and NRAS genes [5,8-10]. Nevertheless, it is important to emphasize that only two of these articles provided data from primary melanomas. In agreement with these earlier reports in primary melanomas with $B R A F$ mutations, we also found a higher frequency of segmental chromosome 7 gain and chromosome 10 loss, which always included $B R A F$ on $7 \mathrm{q} 34$ and PTEN on 10q23.3, which were found to be less frequent in WT tumors or in NRAS mutated lesions. In addition, the group of tumors with $B R A F$ or NRAS mutations also frequently showed deletions in the $9 \mathrm{p}$ chromosomal region, including the loci of $C D K N 2 A$ (9p21.3). Furthermore, we also identified that loss of 11q23.3-11q25 was more frequent in $N R A S^{\text {mut }}$ compared with $B R A F^{\text {mut }}$ tumors. In accordance with previous results, we also frequently detected focal amplification in addition to the activating mutations of the BRAF (7q34) gene. However, in contrast to these previous findings, no correlation was found between the mutation status of the disease and chromosomal loci harboring the TP53 (17p13.1) or CCND1 (11q13) gene, but the loss of one allele at 17p13.3 distal to the TP53 gene was observed frequently in NRAS $S^{\text {mut }}$ melanomas. This second tumor suppressor locus on $17 \mathrm{p}$ has already been observed in brain, breast, lung, and ovarian tumors [34-37]; two candidate tumor suppressor genes HIC1 and OVCA1 were identified at this locus [36,38,39]. It was also suggested that the reduction to hemizygosity of $17 \mathrm{p} 13.3$ resulted in cell cycle deregulation and promoted tumorigenesis in ovarian cancer cells [39].

Using the frequency of high-level amplifications and deletions and relatively conservative statistical methods to highlight regions that differed significantly between tumor subgroups, we found several regions of change more frequently associated with $\mathrm{BRAF}^{\text {mut }}$ or $\mathrm{NRAS}^{\text {mut }}$ primary melanomas than lesions without $B R A F$ or $N R A S$ mutations. Notably, several of the BAC clones that showed greater frequency of loss or gain in one tumor subgroup than another span large genomic regions whose target genes are unknown. In $\mathrm{BRAF}^{\text {mut }}$ melanomas, this pattern is exemplified by the gains and losses involving common tumor-associated regions at the 1q23-25, chromosome 7 and 10q chromosome arm, respectively. This observation is consistent with a previous model that suggests a cooperative effect between the PTEN (10q23.31) and $B R A F(7 \mathrm{q} 34)$ cancer genes in melanoma [40]. It has been implied in several previous studies that the inactivation of the PTEN tumor suppressor gene is a key genetic event in melanoma [41]. In contrast, extended deletions on chromosome 10 suggest a model wherein a broad range of chromosome 10 losses in conjunction with $B R A F$ activation and PTEN inactivation may be involved in polygenic melanoma tumorigenesis. Chromosome 10 deletions are highly prevalent even in early-stage primary melanoma; however, the relative contribution of PTEN inactivation in melanoma progression remains unclear [42]. In addition, alterations mapped within the 11q23q25 locus that carries the well-established tumor suppressor gene named $O P C M L$ were mainly associated with $N R A S^{\text {mut }}$ primary melanoma, indicating another possible link between loss in this region and the consecutive activation of NRAS protein [43]. The observed distinct genetic alterations that are related to the presence or the absence of BRAF or NRAS mutations indicate that there are alternative genetic pathways to melanoma.

The identification of high-level amplifications and homozygous deletions in $\mathrm{BRAF}^{\mathrm{mut}}$ and $\mathrm{NRAS}^{\mathrm{mut}}$ melanomas revealed several regions on $7 \mathrm{p}, 7 \mathrm{q}$, and $13 \mathrm{q}$ chromosome arms that well differentiated these two genotypic subtypes. Here, we highlight three specific regions, in which alterations were frequently observed in other carcinomas as well. The first is the $7 \mathrm{q} 36.3$ region harboring the PTPRN2 gene that was found to be one of the most 
frequent high-level amplifications (14\% of primary melanomas) and was also linked to the presence of $B R A F^{\text {mut }}$. The product of this gene acts as a signaling molecule involved in cell growth, differentiation, mitosis, and oncogenic transformation. In a recent study, the downregulation of a set of genes, including the PTPRN2, was able to suppress metastasis of breast cancer cells to different organ sites [44]. As the second example, high amplification in 6p22.3 has been identified in both BRAF or NRAS mutated melanomas altogether in $16 \%$ of the investigated primary melanomas. The E2F3 candidate gene at this region was already found to be overexpressed in several bladder tumor cell lines with 6p22.3 amplification and knockdown of E2F3 resulted in reduced proliferation of cells [45]. E2F3 is a key repressor of the p19(Arf)-p53 pathway in normal cells [46]. The p19Arf-p53 tumor suppressor pathway plays a critical role in cell-cycle checkpoint control and apoptosis [47]. The third region involves the $17 \mathrm{q} 21.32$ chromosome locus, which was found to be the most frequent homozygous deletion in the investigated primary melanomas ( $23 \%$ of primary melanomas), whose prognostic importance has already been confirmed in primary gastric cancers [48]. HOX genes at this region are reported to be inappropriately expressed in the malignant phenotype, suggesting an involvement of these regulatory proteins in oncogenic transformations [28].

It is well known that genetic events at different genomic regions act concordantly in tumor development. Therefore, we performed a correlation analysis between BAC clones frequently altered in the investigated tumor subgroups to identify regions of chromosomal loss and gain that commonly coexist in $\mathrm{BRAF}^{\text {mut }}$ or WT primary melanomas. It has to be mentioned that our analysis was carried out on a relatively small set of tumors; thus, it only begins to address the possible combinations of cooperating regions. The heat maps on Fig. 3 show trends of correlation between chromosomal regions in the two different melanoma subgroups.

Activation of the EGF/MAPK pathway by genetic alterations is thought to be a main causative factor during melanoma development, and their inhibition sensitizes melanoma cells to certain anticancer agents [49]. Our aim was to explore a relevant subset of gene copy number alterations that have significant impacts on dysregulation of the EGF/MAPK pathway along with the BRAF mutation. A more complete understanding of the genetic alterations that co-occur with mutations of $B R A F$ could help identify therapies that may act synergistically with $B R A F$ kinase inhibitors. The selection of relevant genes for sample classification is one of the challenges in most microarray studies where researchers try to identify the smallest possible set of chromosomal loci or genes that can still achieve good predictive performance. Random forest analysis is increasingly being used in the field of the evaluation of microarray experiments because it has several characteristics that make it ideal to investigate these datasets, such as (a) when the number of variables is much larger than the number of observations and (b) when datasets contain a large number of noisy variables [50-52]. With this method, a particular deletion pattern involving three well-known cancer genes, such as $P T E N, H V H-3$, and $J N K 1$, was identified and found to be associated with the BRAF mutation. PTEN is a wellknown tumor suppressor gene, but little data have been published on the role of $H V H-3$ and JNK1 in melanoma tumorigenesis. According to the literature, the expression of $H V H-3$ resulted in both the specific inactivation and the nuclear translocation of ERK2 [53], and it has been proven that silencing the ERK2 mRNA inhibits tumor growth in vivo [54]. There is also further evidence for the role of the JNK1 gene in tumorigenesis: failure of the function of JNK1 could facilitate tumor formation and $J N K 1^{-} /^{-}$mice developed spontaneous intestinal tumors $[55,56]$. We identified, moreover, a group of other genes to be associated with the $B R A F$ mutation, including SHC1, PEA15, ELK4, EGFR, and PI3K, which have evidence of oncogenic activity or may be potential anticancer treatment targets [16,57]. We also observed that their concomitant amplifications were highly predictive for the BRAF mutation. However, it is worth mentioning that the BAC arrays have a relatively low coverage; thus, we have estimated the copy number changes of each of these pathway genes using the closest BAC clone within $2 \mathrm{Mb}$. Therefore, further experimental follow-up at the expression and interactome level would be needed to determine the exact role of these genes in melanoma carcinogenesis.

Finally, investigating the frequency of the significantly more frequently altered cross-talks among $\mathrm{BRAF}^{\text {mut }}$ and $\mathrm{BRAF}^{\mathrm{WT}}$ melanomas revealed the significant importance of cross-talks between the EGF/MAPK-JAK, EGF/ MAPK-IGF, and JAK-IGF pathways and in the HH pathway in $B R A F$ mutated melanoma progression.

In conclusion, we found marked differences in the genetic pattern of the $B R A F$ or $N R A S$ mutated and WT melanoma subgroups. Therefore, our results also confirm the involvement of distinct genetic pathways in melanoma tumorigenesis that are driven either through $B R A F$ or NRAS mutations. Nevertheless, these observations require further investigation with targeted higher resolution arrays along with studies of mRNA and protein expression to confirm these relevant changes and to yield more effective therapeutic approaches.

\section{Acknowledgements}

This research was supported by the Hungarian National Research Fund (OTKA K75191), the National Research and Development Program, Hungary (NKFP1-00003/ 2005), the Hungarian Academy of Sciences (Grant Number 2006TKI247), and the TÁMOP 4.2.1./B-09/1/ KONV-2010-0007 project; the project is implemented 
through the New Hungary Development Plan, cofinanced by the European Social Fund and the European Regional Development Fund. The authors have no connection with any of the companies or products mentioned in this article.

\section{Conflicts of interest}

There are no conflicts of interest.

\section{References}

1 Leiter U, Garbe C. Epidemiology of melanoma and nonmelanoma skin cancer - the role of sunlight. Adv Exp Med Biol 2008; 624:89-103.

2 Marugame T, Zhang MJ. Comparison of time trends in melanoma of skin cancer mortality (1990-2006) between countries based on the WHO mortality database. Jpn J Clin Oncol 2011; 40:710.

3 Hollestein LM, van den Akker SA, Nijsten T, Karim-Kos HE, Coebergh JW, de Vries E. Trends of cutaneous melanoma in The Netherlands: increasing incidence rates among all Breslow thickness categories and rising mortality rates since 1989. Ann Oncol 2011; 23:524-530.

4 Lens MB, Dawes M. Global perspectives of contemporary epidemiological trends of cutaneous malignant melanoma. Br J Dermato/ 2004; 150 : 179-185

5 Curtin JA, Fridlyand J, Kageshita T, Patel HN, Busam KJ, Kutzner H, et al. Distinct sets of genetic alterations in melanoma. N Engl J Med 2005; 353:2135-2147.

6 Kabbarah OCL. Revealing the genomic heterogeneity of melanoma. Cancer Cell 2005; 8:439-441.

7 Bauer J, Bastian BC. Distinguishing melanocytic nevi from melanoma by DNA copy number changes: comparative genomic hybridization as a research and diagnostic tool. Dermatol Ther 2006; 19:40-49.

8 Greshock J, Nathanson K, Medina A, Ward MR, Herlyn M, Weber BL, et al Distinct patterns of DNA copy number alterations associate with BRAF mutations in melanomas and melanoma-derived cell lines. Genes Chromosomes Cancer 2009; 48:419-428.

9 Gast A, Scherer D, Chen B, Bloethner S, Melchert S, Sucker A, et al. Somatic alterations in the melanoma genome: a high-resolution array-based comparative genomic hybridization study. Genes Chromosomes Cancer 2010; 49:733-745

10 Jonsson G, Dahl C, Staaf J, Sandberg T, Bendahl PO, Ringner M, et al. Genomic profiling of malignant melanoma using tiling-resolution arrayCGH Oncogene 2007; 26:4738-4748.

11 Lazar V, Ecsedi S, Szollosi AG, Toth R, Vizkeleti L, Rakosy Z, et al. Characterization of candidate gene copy number alterations in the $11 \mathrm{q} 13$ region along with BRAF and NRAS mutations in human melanoma. Mod Pathol 2009; 22:1367-1378.

12 Gershenwald JE, Soong SJ, Balch CM. 2010 TNM staging system for cutaneous melanoma... and beyond. Ann Surg Oncol 2010; 17: 1475-1477.

13 Treszl A, Ladanyi A, Rakosy Z, Buczko Z, Adany R, Balazs M. Molecular cytogenetic characterization of a novel cell line established from a superficial spreading melanoma. Front Biosci 2006; 11:1844-1853.

14 Pinkel D, Segraves R, Sudar D, Clark S, Poole I, Kowbel D, et al. High resolution analysis of DNA copy number variation using comparative genomic hybridization to microarrays. Nat Genet 1998; 20:207-211.

15 Jain AN, Tokuyasu TA, Snijders AM, Segraves R, Albertson DG, Pinkel D. Fully automatic quantification of microarray image data. Genome Res 2002; 12:325-332.

16 Rakosy Z, Vizkeleti L, Ecsedi S, Voko Z, Begany A, Barok M, et al. EGFR gene copy number alterations in primary cutaneous malignant melanomas are associated with poor prognosis. Int J Cancer 2007; 121:1729-1737.

17 Rakosy Z, Vizkeleti L, Ecsedi S, Begany A, Emri G, Adany R, et al. Characterization of $9 p 21$ copy number alterations in human melanoma by fluorescence in situ hybridization. Cancer Genet Cytogenet 2008; 182:116-121.

18 Lingjaerde OC, Baumbusch LO, Liestol K, Glad IK, Borresen-Dale AL. $\mathrm{CGH}$-Explorer: a program for analysis of array-CGH data. Bioinformatics 2005; $21: 821-822$.

19 Lai WR, Johnson MD, Kucherlapati R, Park PJ. Comparative analysis of algorithms for identifying amplifications and deletions in array $\mathrm{CGH}$ data. Bioinformatics 2005; 21:3763-3770.

20 Tusher VG, Tibshirani R, Chu G. Significance analysis of microarrays applied to the ionizing radiation response. Proc Natl Acad Sci USA 2001; 98: 5116-5121.
21 Peter H, Westfall SSY. Resampling-based multiple testing: examples and methods for $P$ value adjustment. New York: John Wiley and Sons; 1993.

22 Loo LW, Grove DI, Williams EM, Neal CL, Cousens LA, Schubert EL, et al. Array comparative genomic hybridization analysis of genomic alterations in breast cancer subtypes. Cancer Res 2004; 64:8541-8549.

23 Hornberg JJ, Bruggeman FJ, Westerhoff HV, Lankelma J. Cancer: a systems biology disease. Biosystems 2006; 83:81-90.

24 Stelling J, Sauer U, Szallasi Z, Doyle FJ III, Doyle J. Robustness of cellular functions. Cell 2004; 118:675-685.

25 Korcsmaros T, Farkas IJ, Szalay MS, Rovo P, Fazekas D, Spiro Z, et al. Uniformly curated signaling pathways reveal tissue-specific cross-talks and support drug target discovery. Bioinformatics 2010; 26:2042-2050.

26 Liaw AWM. Classification and regression by randomForest. R News 2002; 2:18-22.

27 Breiman L. Random forests. Machine Learning 2001; 45:5-32.

28 Raman V, Martensen SA, Reisman D, Evron E, Odenwald WF, Jaffee E, et al. Compromised HOXA5 function can limit p53 expression in human breast tumours. Nature 2000; 405:974-978.

29 Bastian BC, Olshen AB, LeBoit PE, Pinkel D. Classifying melanocytic tumors based on DNA copy number changes. Am J Pathol 2003; 163:1765-1770.

30 Wiltshire RN, Dennis TR, Sondak VK, Meltzer PS, Trent JM. Application of molecular cytogenetic techniques in a case study of human cutaneous metastatic melanoma. Cancer Genet Cytogenet 2001; 131:97-103.

31 Balazs M, Adam Z, Treszl A, Begany A, Hunyadi J, Adany R. Chromosoma imbalances in primary and metastatic melanomas revealed by comparative genomic hybridization. Cytometry 2001; 46:222-232.

32 Ribas A, Flaherty KT. BRAF targeted therapy changes the treatment paradigm in melanoma. Nat Rev Clin Oncol 2011; 8:426-433.

33 Sebolt-Leopold JS, Herrera R. Targeting the mitogen-activated protein kinase cascade to treat cancer. Nat Rev Cancer 2004; 4:937-947.

34 Tsuchiya E, Tanigami A, Ishikawa Y, Nishida K, Hayashi M, Tokuchi Y, et al. Three new regions on chromosome 17p13.3 distal to p53 with possible tumor suppressor gene involvement in lung cancer. Jpn J Cancer Res 2000; 91:589-596.

35 Chattopadhyay P, Rathore A, Mathur M, Sarkar C, Mahapatra AK, Sinha S Loss of heterozygosity of a locus on $17 \mathrm{p} 13.3$, independent of $\mathrm{p} 53$, is associated with higher grades of astrocytic tumours. Oncogene 1997; 15:871-874.

36 Parrella P, Scintu M, Prencipe M, Poeta ML, Gallo AP, Rabitti C, et al. HIC1 promoter methylation and 17 p13.3 allelic loss in invasive ductal carcinoma of the breast. Cancer Lett 2005; 222:75-81.

37 Phillips NJ, Ziegler MR, Radford DM, Fair KL, Steinbrueck T, Xynos FP, et al Allelic deletion on chromosome $17 \mathrm{p} 13.3$ in early ovarian cancer. Cancer Res 1996; 56:606-611.

38 Schultz DC, Vanderveer L, Berman DB, Hamilton TC, Wong AJ, Godwin AK. Identification of two candidate tumor suppressor genes on chromosome 17p13.3. Cancer Res 1996; 56:1997-2002.

39 Bruening W, Prowse AH, Schultz DC, Holgado-Madruga M, Wong A, Godwin AK. Expression of OVCA1, a candidate tumor suppressor, is reduced in tumors and inhibits growth of ovarian cancer cells. Cancer Res 1999; 59:4973-4983.

40 Tsao H, Goel V, Wu H, Yang G, Haluska FG. Genetic interaction between NRAS and BRAF mutations and PTEN/MMAC1 inactivation in melanoma. J Invest Dermatol 2004; 122:337-341.

$41 \mathrm{Wu} \mathrm{H}$, Goel V, Haluska FG. PTEN signaling pathways in melanoma Oncogene 2003; 22:3113-3122.

42 Lin WM, Baker AC, Beroukhim R, Winckler W, Feng W, Marmion JM, et al. Modeling genomic diversity and tumor dependency in malignant melanoma. Cancer Res 2008; 68:664-673.

43 Sellar GC, Watt KP, Rabiasz GJ, Stronach EA, Li L, Miller EP, et al. OPCML at $11 \mathrm{q} 25$ is epigenetically inactivated and has tumor-suppressor function in epithelial ovarian cancer. Nat Genet 2003; 34:337-343.

44 Tavazoie SF, Alarcon C, Oskarsson T, Padua D, Wang Q, Bos PD, et al. Endogenous human microRNAs that suppress breast cancer metastasis. Nature 2008; 451:147-152.

45 Huang L, Luo J, Cai Q, Pan Q, Zeng H, Guo Z, et al. MicroRNA-125b suppresses the development of bladder cancer by targeting E2F3. Int J Cancer 2011; 128:1758-1769.

46 Aslanian A, laquinta PJ, Verona R, Lees JA. Repression of the Arf tumor suppressor by E2F3 is required for normal cell cycle kinetics. Genes Dev 2004; 18:1413-1422.

47 Levine AJ. p53, the cellular gatekeeper for growth and division. Cell 1997; 88:323-331.

48 Tomioka N, Morita K, Kobayashi N, Tada M, Itoh T, Saitoh S, et al. Array comparative genomic hybridization analysis revealed four genomic 
prognostic biomarkers for primary gastric cancers. Cancer Genet Cytogenet 2010; 201:6-14.

49 Sinnberg T, Lasithiotakis K, Niessner H, Schittek B, Flaherty KT, Kulms D, et al. Inhibition of PI3K-AKT-mTOR signaling sensitizes melanoma cells to cisplatin and temozolomide. J Invest Dermatol 2009; 129:1500-1515.

50 Diaz-Uriarte R, Alvarez de Andres S. Gene selection and classification of microarray data using random forest. BMC Bioinformatics 2006; 7:3-16.

51 Gunther EC, Stone DJ, Gerwien RW, Bento P, Heyes MP. Prediction of clinical drug efficacy by classification of drug-induced genomic expression profiles in vitro. Proc Natl Acad Sci USA 2003; 100:9608-9613.

52 Alvarez S, Diaz-Uriarte R, Osorio A, Barroso A, Melchor L, Paz MF, et al. A predictor based on the somatic genomic changes of the BRCA1/BRCA2 breast cancer tumors identifies the non-BRCA1/BRCA2 tumors with BRCA1 promoter hypermethylation. Clin Cancer Res 2005; 11: 1146-1153.
53 Mandl M, Slack DN, Keyse SM. Specific inactivation and nuclear anchoring of extracellular signal-regulated kinase 2 by the inducible dual-specificity protein phosphatase DUSP5. Mol Cell Biol 2005; 25:1830-1845.

54 Bessard A, Fremin C, Ezan F, Fautrel A, Gailhouste L, Baffet G. RNAimediated ERK2 knockdown inhibits growth of tumor cells in vitro and in vivo. Oncogene 2008; 27:5315-5325.

55 Ahmed SU, Milner J. Basal cancer cell survival involves JNK2 suppression of a novel JNK1/c-Jun/Bcl-3 apoptotic network. PLoS One 2009; 4: e7305-e7318.

56 Tong C, Yin Z, Song Z, Dockendorff A, Huang C, Mariadason J, et al. c-Jun $\mathrm{NH} 2$-terminal kinase 1 plays a critical role in intestinal homeostasis and tumor suppression. Am J Pathol 2007; 171:297-303.

57 Hu L, Zaloudek C, Mills GB, Gray J, Jaffe RB. In vivo and in vitro ovarian carcinoma growth inhibition by a phosphatidylinositol 3-kinase inhibitor (LY294002). Clin Cancer Res 2000; 6:880-886. 


\section{AUTHOR QUERY FORM \\ LIPPINCOTT WILLIAMS AND WILKINS}

JOURNAL NAME: CMR

ARTICLE NO: $\quad 200515$

QUERIES AND / OR REMARKS

\begin{tabular}{|l|l|l|}
\hline QUERY NO. & \multicolumn{1}{|c|}{ Details Required } & Author's Response \\
\hline & No queries & \\
\hline
\end{tabular}

